\title{
Effects of Levosimendan on cardiac function, size and strain in heart failure patients
}

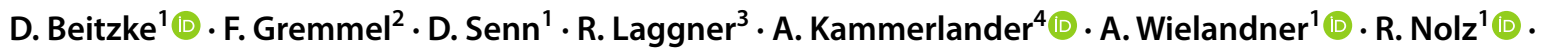 \\ M. Hülsmann ${ }^{4}$. C. Loewe ${ }^{1} \mathbb{D}$
}

Received: 21 July 2020 / Accepted: 16 October 2020 / Published online: 24 October 2020

(c) The Author(s) 2020

\begin{abstract}
Levosimendan improves cardiac function in heart failure populations; however, its exact mechanism is not well defined. We analysed the short-term impact of levosimendan in heart failure patients with ischemic and non-ischemic cardiomyopathy (CMP) using multiparametric cardiac magnetic resonance (CMR). We identified 33 patients with ischemic or non-ischemic CMP who received two consecutive CMR scans prior to and within one week after levosimendan administration. Changes in LV ejection fraction (LVEF) and LV volumes, as well as changes in strain rates, were measured prior to and within one week after levosimendan infusion. LV scarring, based on late gadolinium enhancement (LGE), was correlated to changes in LV size and strain rates. Both LV endiastolic (EDV) and endsystolic volumes (ESV) significantly decreased (EDV: $p=0,001$; ESV: $p=0,002)$ after levosimendan administration, with no significant impact on LVEF $(p=0.41)$, cardiac output ( $p=0.61)$, and strain rates. Subgroup analyses of ischemic or non-ischemic CMP showed no significant differences between the groups in terms of short-term LV reverse remodeling. The presence and extent of scarring in LGE did not correlate with changes in LV size and strain rates. CMR is able to monitor cardiac effects of levosimendan infusion. Short-term follow-up of a single levosimendan infusion using CMR shows a significant decrease in LV size, but no impact on LVEF or strain measurements. There was no difference between patients with ischemic or non-ischemic CMP. Quantification of LV scarring in CMR is not able to predict changes in LV size and strain rates in response to levosimendan.
\end{abstract}

Keywords Heart failure $\cdot$ Cardiac magnetic resonance $\cdot$ Strain imaging $\cdot$ Levosimendan

\section{Introduction}

The inodilator levosimendan is a calcium sensitizer used in patients with acute, decompensated heart failure or in the setting of cardiac surgery for ischemic heart disease or valvular heart disease [1]. In addition to the positive inotropic effects, anti-inflammatory, anti-oxidant, and cardioprotective

D. Beitzke

dietrich.beitzke@meduniwien.ac.at

1 Department of Biomedical Imaging and Image-guided Therapy, Medical University of Vienna, Waehringer Guertel 18-20, 1090 Vienna, Austria

2 Department of Surgery, Medical University of Vienna, Vienna, Austria

3 Department of Orthopedics and Trauma Surgery, Medical University of Vienna, Vienna, Austria

4 Department of Internal Medicine II / Division of Cardiology, Medical University of Vienna, Vienna, Austria effects are presumed that directly affect the heart [2]. On top of these cardiac effects, the peripheral resistance is lowered as a consequence of levosimendan-induced vasodilation [2]. Which system - the heart or the peripheral system-is predominantly affected is still a matter of debate, and therefore, the use of levosimendan in acute heart failure patients remains controversial. Some prospective studies favor drug usage, whereas other randomized trials do not support the advantages of levosimendan therapy [1, 3-5]. An overall definitive advantage of levosimendan administration is lacking, but there might be subgroups of patients who would benefit from its use [6]. Predictors of hemodynamic response to drug exposure also remain unclear [7].

Cardiac magnetic resonance (CMR) is a well-established tool for the assessment of cardiac function und structure. CMR is considered the gold standard in the assessment of ventricular function and allows for accurate scar quantification based on late gadolinium enhancement (LGE) [8]. The presence or absence of scarring in non-ischemic dilated 
cardiomyopathy (CMP), as well as quantification of infarct size and transmurality in ischemic cardiomyopathy (ICMP) , is already an independent risk factor for treatment success in case of revascularization $[9,10]$. The presence of LGE is also associated with the decreased ability for reverse remodeling in LV dilatation [11].

Recent advances in CMR feature tracking techniques also allow for the evaluation of myocardial deformation. The technique is based on identifying the motion of cardiac structures through the cardiac cycle. From the assessment of these movements, longitudinal and circumferential strain values can be derived that provide information about myocardial fiber function [12]. Here, global longitudinal strain (GLS) measurements are more representative of subendocardial fiber function, whereas global circumferential strain (GCS) measurements are more representative of subepicardial fiber function [12]. The technique has already undergone validation in terms of reproducibility, and reduced GLS (derived by echocardiography and CMR) has also been proven to be an independent risk factor in patients with dilated cardiomyopathy (DCM) $[13,14]$. Furthermore, strain measurements could allow for monitoring of drug interventions or revascularization procedures [15].

Therefore, the aim of this analysis was to evaluate the impact of levosimendan on cardiac function, structure, and deformation, assessed CMR in a heart failure population.

\section{Materials and methods}

All patients who received levosimendan prior to planned heart surgery for valvular and/or coronary artery bypass grafting between March 2010 and February 2018 were identified via the institutional pharmacy records. This cohort was scanned for the presence of two consecutive CMR scans that were obtained during the clinical routine work-up for the evaluation of LV function, size, viability assessment and presence of scarring.

Overall, 54 patients were identified who received levosimendan in the analysis period. Seven patients were excluded due to the lack of two consecutive CMR (three patients with a pacemaker, four patients with other contraindications for CMR). Another five patients were excluded due to right heart failure based on pulmonary or tricuspid valve insufficiency.

\section{CMR}

All patients were scanned on a $1.5 \mathrm{~T}$ system (Siemens Avanto Fit; Siemens Healthineers; Erlangen; Germany). The CMR imaging protocol included steady state free precession (SSFP) imaging in two-, three-, and four-chamber views, using the left ventricular outflow tract and short axis for the evaluation of cardiac function (TR $3.2 \mathrm{~ms}$, TE $1.2 \mathrm{~ms}$; flip angle, $64^{\circ}$; voxel size, $1.4 \times 1.4 \times 36 \mathrm{~mm}$; matrix $180 \times 256$ pixels, slice thickness $8 \mathrm{~mm}$ ). Phase contrast angiography was performed in patients with aortic insufficiency in slices perpendicular to the ascending aorta at the sinotubular junction. Typical parameters were: TR: $4.8 \mathrm{~ms}$; TE: $2.8 \mathrm{~ms}$; matrix: $320 \times 300 \mathrm{~mm}$; flip angle: $12^{\circ}$; temporal resolution: 25 to $55 \mathrm{~ms}$; and velocity window: 2.5 to $4.0 \mathrm{~m} / \mathrm{s}$ depending on the presence or absence of alaising. LGE was performed in the short axis, with two-, three-, and four-chamber views, 10 to 15 minutes after the injection of $0.15 \mathrm{ml}$ gadobutrol (Gadovist ${ }^{\circledR}$, Bayer-Schering, Austria) per kg bodyweight using T1-weighted, phase-sensitive, inversion-recovery (PSIR) sequences (TR: $849.6 \mathrm{~ms}$; TE:1.07ms; voxel size $1.4 \times 1.4 \times 3.8 \mathrm{~mm}$; $146 \times 256$ matrix, slice thickness $8 \mathrm{~mm}$; distance factor $0 \%$ ). A second CMR scan without LGE was conducted after the administration of levosimendan.

\section{CMR postprocessing}

For CMR evaluation, $\mathrm{LV}$ volumes and function were derived from short axis Cine SSFP, whereas scarring was determined from the LGE images. To quantify both functional and scar parameters, a commercially available post-processing software was used (QMass, Medis; Leiden, NL). LV function was derived from short axis Cine SSFP images in diastole and systole according to valid recommendations [16]. Positive LGE was analyzed using the full width at half maximum method [17]. Regurgitant fraction in patients suffering from aortic insufficiency was analysed by placing a region of interest at the vessel borders of through plane phase contrast angiography [16].

Forty-two patients were included in the advanced CMR postprocessing analysis (QStrain, Medis; Leiden, NL). For the assessment of global longitudinal strain (GLS) and global circumferential strain (GCS), long axis and short axis Cine SSFP were analyzed using a recently developed feature-tracking software (QStrain, Medis, Leiden, NL). During the analysis, another nine patients had to be excluded due to poor image quality/motion artifacts $(n=4)$, lack of LGE $(n=1)$, or incompatibility of the CMR scans with the featuretracking postprocessing software $(n=4)$.

\section{Patient population}

Ultimately, 33 patients were included for analysis. Patient characteristics are listed in Table 1. All patients received standard heart failure therapy (Table 1).

ICMP, defined by the presence of subendocardial LGE, was present in 17 patients (52\%), and valvular disease was 
Table 1 Clinical characteristics of patients receiving levosimendan

\begin{tabular}{ll}
\hline Demographics & \\
\hline Male Sex (n) & $30(91 \%)$ \\
Age (years) & $62 \mathrm{a}(50-79 \mathrm{a})$ \\
Risk factors & $\mathrm{n}(\%)$ \\
Hypertension & $30(91)$ \\
Diabetes mellitus & $13(39)$ \\
Hyperlipidemia & $19(58)$ \\
(History of) Smoking & $15(45)$ \\
BMI >25 & \\
Etiology of heart failure & $\mathrm{n}(\%)$ \\
Ischemic cardiomyopathy & $17(52)$ \\
Valvular disease & $10(30)$ \\
Ischemic cardiomyopathy and valvular disease & $6(18)$ \\
Medication & \\
Beta blocker & $30(91)$ \\
ACE/ATII Inhibitors & $32(97)$ \\
Spironolactone & $29(88)$ \\
Diuretics & $16(48)$ \\
Digoxin & $1(3)$ \\
\hline
\end{tabular}

observed in 10 patients $(30 \%)$. In the latter group, three patients presented with severe aortic stenosis, four patients presented with aortic insufficiency, two patients had an isolated mitral valve insufficiency, and one patient presented with a combination of an aortic stenosis and a severe mitral valve insufficiency. A combination of ICMP and valvular disease was observed in six patients (18\%; aortic stenosis $\mathrm{n}=2$; aortic insufficiency $\mathrm{n}=2$; mitral valve insufficiency $\mathrm{n}=2$ ). In patients with aortic insufficiency mean phase contrast derived regurgitant fraction was $42.5 \%$ (SD $17.8 \%$; range $14-72 \%$ ).

\section{Levosimendan administration}

Levosimendan was administered via a $24 \mathrm{~h}$ lasting infusion $(0.1 \mathrm{mcg} / \mathrm{kg} / \mathrm{min})$ without the application of a starting bolus under hemodynamic monitoring in an intermittent care setting. Brain natriuretic peptide levels were assessed before and 3 days after the infusion.

\section{CMR post levosimendan}

Three to seven (mean 3.8) days after levosimendan infusion, a second CMR without LGE was performed to evaluate changes in LV size, function, and strain. All analyses were performed by experienced CMR readers (RL, DS), in consensus, strictly according to the recommendations of the society of cardiac magnetic resonance [16].
Consecutive scans from each patients were analysed within a single session.

\section{Statistical analysis}

All statistical analyses were performed using SPSS for Windows (version 23.0; IBM Corporation, Somers, NY, USA). Baseline characteristics are displayed as mean $\pm \mathrm{SD}$, or median (IQR), and total numbers (\%), as appropriate. Normal distributed parameters of LV function, size, and strain parameters were compared using a paired T-test. Non-normally distributed parameters (NT-proBNP) were compared using the paired Wilcoxon test.

We stratified patients according to the presence or absence of post-ischemic scarring on LGE, defined by the presence of a subendocardial scar and a scar volume of more than $5 \%$ of the LV mass. In this subgroup analysis, not only the absolute values of the LV parameters, but also the delta values of the parameters were compared between the groups using a univariate ANOVA. A subgroup comparison of NT-proBNP was performed using the Kruskal-Wallis test. Furthermore, the potential impact of scar volume on changes in LV function was assessed by a correlating the LV scar volume (\%) to the $\Delta$ of LV functional parameters using a Pearson correlation.

\section{Results}

\section{Impact of levosimendan on LV function}

Mean end diastolic volume (EDV), and mean end systolic volumes (ESV) significantly decreased after levosimendan infusion (mean EDV before levosimendan $259.8 \pm 87.6$ $\mathrm{ml}$ to $245.1 \pm 87.7 \mathrm{ml}$ [103.63-437.7] after the infusion $-\mathrm{p}=0.001$; mean ESV before levosimendan $187.1 \pm 68$ decreased to $174 \pm 71.6 \mathrm{ml}$ [54.6-343.9] $-\mathrm{p}=0.002$ ) (Fig. 1). All other CMR-derived LV functional parameters did not change significantly (stroke volume [SV] from $72.7 \pm 33.5 \mathrm{ml}$ to $71.1 \pm 33.1 \mathrm{ml}$; ejection fraction from $28.3 \pm 8.8 \%$ to $29.2 \pm 9.4 \%$ before and after infusion, respectively). Cardiac output $(\mathrm{CO})\left(5 \pm 2.31 / \mathrm{m}^{2}\right.$ to $\left.5.1 \pm 2.31 / \mathrm{m}^{2}\right)$, global longitundinal strain (GLS) $(-7.8 \pm$ $4 \%$ to $-8.3 \pm 4 \%$ ), global circumferential strain (GCS) $(-12.3 \pm 5.5 \%$ to $-12.4 \pm 5.6 \%)$, and global radial strain (GRS) $(16.7 \pm 8.9$ to $17.6 \pm 8.8)$ also remained unaffected Results are shown in Table 2.

NT-proBNP significantly decreased from a of mean $5778.5 \pm 7623.5 \mathrm{pg} / \mathrm{ml}$ to $4017.9 \pm 5652.4 \mathrm{pg} / \mathrm{ml}(\mathrm{p}=$ $0.002)$. 


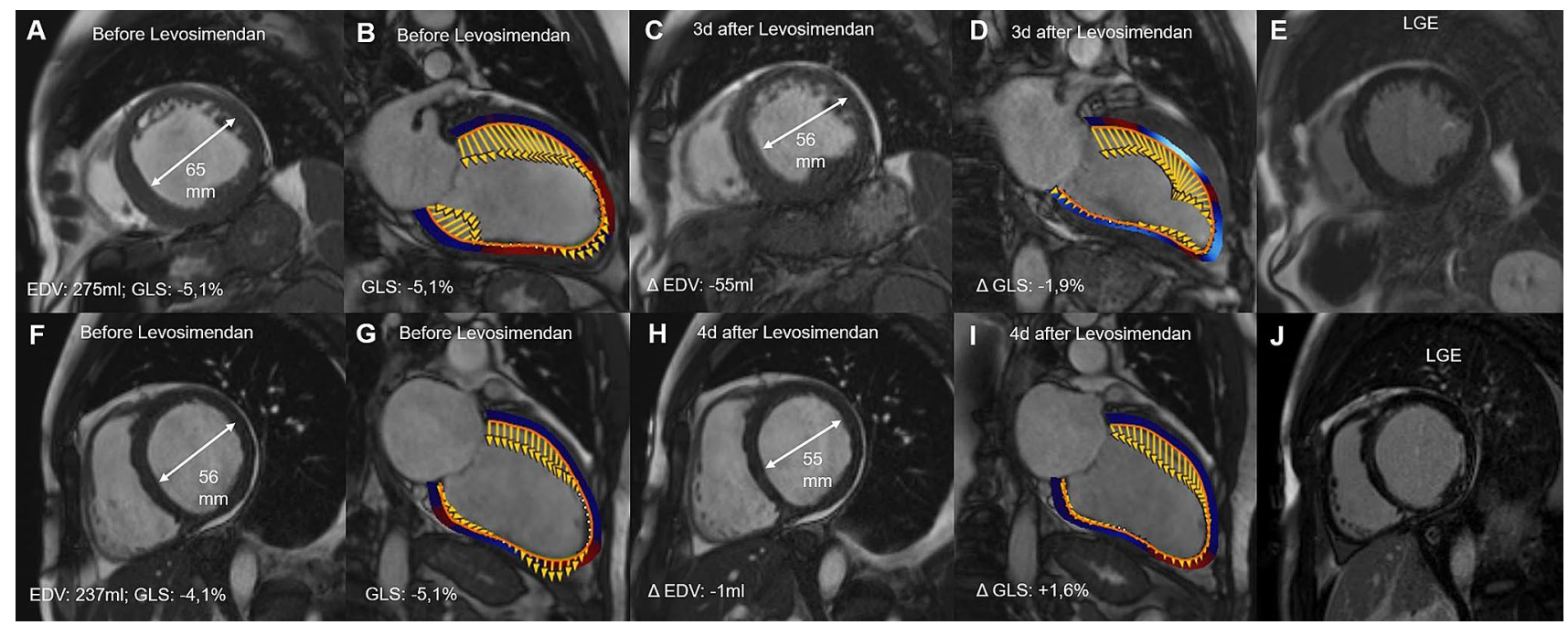

Fig. 1 CMR before and after Levosimendan infusion.b-e shows a decrease in LV size after Levosimendan accompanied by an improvement in strain rate in a patient with aortic insufficiency and no evidence for LV scarring.

Table 2 Results for left ventricular volumetry, ejection fraction, strain parameters, and brain natriuretic peptide levels prior to and after levosimendan infusion

\begin{tabular}{llll}
\hline LV Parameter & Pre levosimendan & Post levosimendan & $\mathrm{p}$ \\
\hline EDV $(\mathrm{ml})$ & $259.8 \pm 87.6[113.3-457.6]$ & $245.1 \pm 87.7[103.63-437.7]$ & $\mathbf{0 . 0 0 1}$ \\
ESV $(\mathrm{ml})$ & $187.1 \pm 68[72.7-339.1]$ & $174 \pm 71.6[54.6-343.9]$ & $\mathbf{0 . 0 0 2}$ \\
SV $(\mathrm{ml})$ & $72.7 \pm 33.5[28.6-168.6]$ & $71.1 \pm 33.1[22.4-159.5]$ & 0.59 \\
EF $(\%)$ & $28.3 \pm 8.8[12.9-48.6]$ & $29.2 \pm 9.4[12.9-47.3]$ & 0.41 \\
CO $(1 / \mathrm{min})$ & $5 \pm 2.3[2.1-11.6]$ & $5.1 \pm 2.3[2.2-11.7]$ & 0.61 \\
GLS & $-7.8 \pm 4[-17.9$ to -0.82$]$ & $-8.3 \pm 4[-14.9$ to -1.4$]$ & 0.09 \\
GCS & $-12.3 \pm 5.5[-27.1-0.27]$ & $-12.4 \pm 5.6[-27$ to -2.7$]$ & 0.69 \\
GRS & $16.7 \pm 8.9[2.7-45.1]$ & $17.6 \pm 8,8[4.1-38.6]$ & 0.14 \\
NT-proBNP ng/ml & $5778.5 \pm 7623.5[432-35000]$ & $4017.9 \pm 5652.4[121.9-25533]$ & $\mathbf{0 . 0 0 2}$ \\
LGE $(\%$ of LV) & $16.9 \pm 11.9[0-45,9]$ & & N/A \\
\hline
\end{tabular}

$L V$ left ventricle, $E D V$ endiastolic volume, $E S V$ endsystolic volume, $S V$ troke volume, $E F$ ejection fraction, $C O$ cardiac output, $G L S$ global longitudinal strain, GCS global cirumferential strain, GRS global radial strain, NT pro BNP N-terminal pro brain natriuretic peptide, $L G E$ late gadolinium enhancement

\section{Group comparison of patients with and without Ischemic scar burden}

Twenty-three patients presented with subendocardial scars (ICMP), and/or a combination of ICMP and valvular disease. Ten patients showed LV dilatation based on valvular disease only, but without any post-ischemic, subendocardial scar. Results of a group comparison of these two patient cohorts is shown in Table 3. In patients with ICMP, GLS was significantly lower $(6.8 \pm 4$ vs. $-9.9 \pm$ $3.3 ; \mathrm{p}=0.04)$ prior to levosimendan administration. After levosimendan, this significance vanished. The $\Delta$ for GLS changes between the groups showed no significant difference. No other parameters showed any difference between the groups prior to or after levosimendan infusion.

\section{Correlation between LV scarring and changes in LV function}

Table 4 shows the results from the Pearson correlation between the percentage of LV scarring and changes in LV size, function, and strain. Overall, the percentage of LV scar in the study population showed no correlation to the changes in the functional parameters nor to the changes in LV size.

\section{Discussion}

In this retrospective analysis using multiparametric CMR, we were able to monitor the response to levosimendan infusion in patients with systolic heart failure. In the study cohort, a single dose of levosimendan resulted in significant reduction in LV size, shown by a significant decrease 


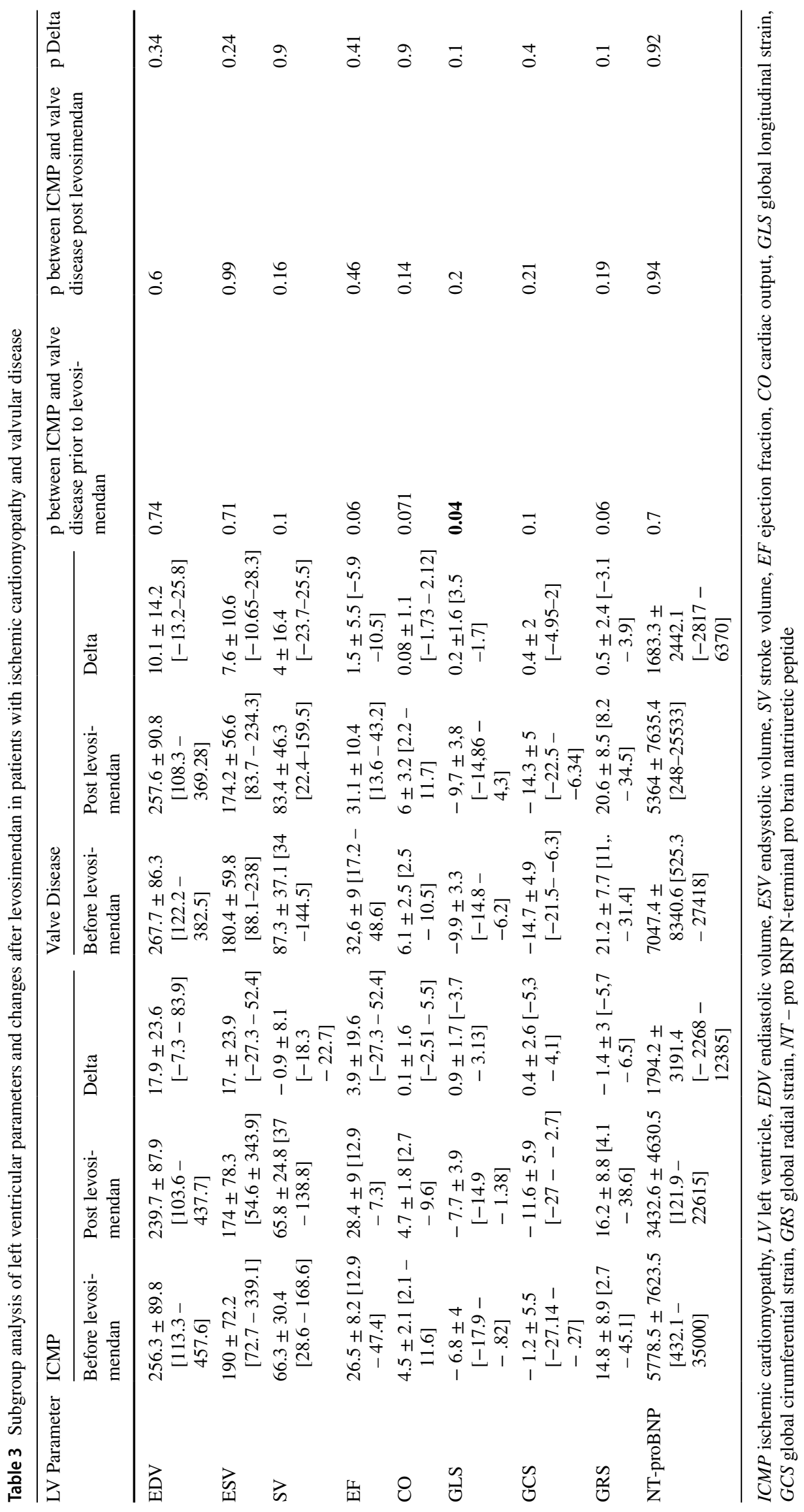


Table 4 Correlation between changes in left ventricular parameters with cardiac magnetic resonance and the percentage of left ventricular scar load by late gadolinium enhancement

\begin{tabular}{llcl}
\hline & $\Delta$ & Pearson $\mathrm{r}$ & $\mathrm{p}$ \\
\hline Scar $\%$ & $\Delta$ EDV & 0.21 & 0.25 \\
& $\Delta$ ESV & $0 . .24$ & 0.17 \\
& $\Delta$ SV & 0.13 & 0.45 \\
& $\Delta$ EF & $0 . .08$ & 0.67 \\
$\Delta$ CO & 0.07 & 0.71 \\
& $\Delta$ GLS & -0.004 & 0.98 \\
& $\Delta$ GCS & -0.06 & 0.73 \\
& $\Delta$ GRS & 0.03 & 0.89 \\
& $\Delta$ NT-pro BNP & -0.16 & 0.374 \\
\hline
\end{tabular}

$E D V$ endiastolic volume, $E S V$ endsystolic volume, $S V$ stroke volume, $E F$ ejection fraction, $C O$ cardiac output, $G L S$ global longitudinal strain, GCS global cirumferential strain, GRS global radial strain, $N T$-pro BNP N-terminal pro brain natriuretic peptide

in LVEDV and LVESV (Fig 1). This resulted in unchanged LVEF values, as well as a non- significant increase in CO.. Most importantly, in this cohort, we could not show a significant increase in deformity parameters that might be representative of the positive inotropic effects of the drug on the LV myocardium using CMR strain imaging. Despite the fact that baseline GLS was significantly reduced in patients with ICMP when compared to patients with valvular disease, levosimendan infusion had no effect on LV strain values. Furthermore, the absolute amount of LV scarring assessed by LGE showed no correlation to changes in LV size, function, and strain after levosimendan infusion. Therefore, the amount of LV scaring seems to have no clinical impact on short-term treatment response to levosimendan infusion.

Levosimendan for the treatment of acute heart failure has been shown to be safe and efficient compared to placebo and dobutamine $[1,18]$. However, its usefulness in the preor post-operative setting in in patients with low EF and/or conditions of acute heart failure in clinical practice remains controversial, as randomized studies have shown no impact on the length of intensive care unit stay or mortality $[3,4]$. Therefore, appropriate indications and patient selection, as well as timing of the infusion in the setting of potential cardiac surgery, might be of clinical importance [6]. From available imaging modalities to date, only echocardiography has been used to monitor LV function with the use of the drug $[19,20]$. With the exception of a case report, CMR, including tissue characterization for treatment monitoring, has not been reported in the literature thus [21].

In our cohort, using CMR, treatment response to levosimendan resulted in a significant decrease in LV size after a single infusion over 24 hours. This resulted in only a nonsignificant change in LV EF. These findings with CMR in our cohort differ from data reported from echocardiograms or studies using pulmonary catheterization [1, 22, 23]. This might be based on the difference in the evaluation of the EF primarily when using non-enhanced $2 \mathrm{D}$ echocardiography [24]. For example, Navarri et al. showed significant changes after levosimendan infusion in LVEF with echocardiography, but, at the same time, nonsignificant changes in LV systolic and LV diastolic values [22]. These finding are in contrast to ours and may be based on the methodology used for assessing LV volumes and LV EF. Imaging reports showed significantly lower EF values in echocardiography when comparing unenhanced echocardiography to CMR, and, despite a good correlation between the modalities, a wide limit of agreement in heart failure patients, especially with regard to LV volumes $[25,26]$. This might favor the use of CMR in heart failure cohorts with severely dilated LVs. Furthermore, we were not able to exactly compare our study cohort to others published in the literature in terms of LV volumes, as most of the studies used invasive pulmonary artery catheterization to report on SV or solely reported on outcomes.

CMR, including LGE for quantification of scar load, has not been applied to response assessment or response prediction after levosimendan administration. In the literature, mostly invasive monitoring using pulmonary artery catheterization or echocardiography has been reported $[1,20]$. In this cohort, LGE was assessed for myocardial viability or for diagnostic proposes in the assessment of LV dilatation $[9,10]$. We evaluated whether there was an influence of scar load on therapy response to levosimendan. The aim of this analyses was to screen for patients who might not profit from levosimendan because of high (ischemic or nonischemic) scar burden, as this would have an impact on costs and might avoid potential complications of levosimendan infusion, such as hypotension and hypokalemia. However, in our cohort, we were not able to show a correlation between changes in LV parameters and the percentage of scar load. In addition, there was no difference between patients with known ischemic CMP and dilatative CMP based on the presence of valvular disease. These findings are similar to results published by Najjar et al., who showed positive hemodynamic effects of levosimendan in a heart failure cohort, but failed to identify predictors of the response to drug exposure [7]. Prospective studies are lacking about the influence of LV structure on treatment response or even outcomes.

The assessment of left ventricular strain parameters and changes in these parameters appear to be valuable predictors of treatment response in various cardiac diseases [12]. The methodology has already shown proven reproducibility with an acceptable inter- and intraobserver variability [14]. Therefore, strain measurements seem to be a valuable alternative to EF measurements, as these parameters might be more representative of the improvement in myocardial function and deformity than EF measurements based on the ratio between 
EDV and ESV alone [27]. Strain measurements, especially GLS, have already been proven as an independent predictor of outcome in CMP and non-ischemic DCM, showing that a reduction in strain of one standard deviation has a severe impact on the hazard ratio for cardiovascular events [28, 29]. In ischemic heart disease GLS and GCS changes in the early phase after myocardial infarction were predictive of outcome [15]. More data on changes in strain measurements after medical or vascular interventions are currently lacking in the literature. Despite significant changes in LV volumes after levosimendan infusion, no changes in CMR-derived strain parameters could be observed in our cohort. Therefore, the observed reduction in LV size in our cohort might be based solely on lowering the peripheral resistance and not based on changes in inotropy. Dalla et al. described in his model with septic patients receiving norepinephrine that changes in afterload and preload i.e. an increase in systemic and pulmonary vascular resistance, impact LV EDV, but not ejection fraction or longitudinal strain [30]. The lack of changes in strain parameters in this cohort could also be explained by a lack of ability in the improvement of deformity based on longstanding LV dilatation, presence of valvular disease, and the presence of fibrosis. The mean percentage of LV fibrosis was $16 \%$ in our study group and it has already been shown that the presence of LGE is associated with reduced strain parameters [14]. Furthermore, the presence of LGE had an impact on GLS values prior to levosimendan infusion in our ICMP subgroup. However, we could not prove a correlation between the absolute amount of scarring by LGE and the changes in GLS in this subgroup, and strain parameters numerically showed even larger changes in the ICMP group when compared to valvular disease. Therefore, LGE does not predict (but also does not prevent) treatment response to levosimendan infusion.

In DCM, imaging studies on short-term changes in LV size are lacking and results of long-term studies are somehow divergent. On the one hand, the presence of LGE has been shown to be a negative predictor of LV reverse remodeling in non-ischemic CMP [31]. On the other hand, Tayal et al. failed to identify the measurement of cardiac structure or cardiac strain as a marker for LV LV recovery in DCM [32]. In their work, contractile reserve, as evidenced by dobutamine stress echocardiography, was predictive for a reduction in LV size [32]. In our cohort, we observed significantly smaller LV volumes after levosimendan infusion regardless of the presence of LV scarring in both groups.

There are several limitations of our study. First, it is a retrospective, single-center cohort study with the focus on imaging parameters. Data on the outcome and/or impact of levosimendan infusion on intermediate and long-term outcome are not available. In addition, the sample size is small and patients were selected non-randomly by a single referring physician, which may have caused a patient selection bias for the analysis. On the other hand by this patient selection was more homogenous than in a multi center setting. Additionally data on hemodynamic changes in case of valvular disease (e.g. changes in regurgitant fraction) are missing as flow measurements have not been implemented in all CMR studies.

\section{Conclusion}

Multiparametric CMR including strain imaging can be used to monitor effects of levosimendan infusion. In patients with ischemic or non-ischemic CMP, a significant reduction in LV size can be observed after a single dose of levosimendan. This was not accompanied by a significant change in LV deformity parameters evaluated by strain measurements. CMR imaging and quantification of myocardial scarring is not able to predict treatment response to a single dose of levosimendan.

F-J shows a patients with ischemic cardiomyopathy and a subendocardial scar in the lateral wall. CMR before and after levosimendan infusion shows no decrease in LV size and no changes in global longitudinal strai

Funding Open access funding provided by Medical University of Vienna.

\section{Compliance with ethical standards}

Conflict of interest All authors declare that they have no conflict of interest.

Ethical approval Data analyses were approved by the local institutional review board. In accordance with the review board, informed consent was waived due to the retrospective nature of this analysis.

Open Access This article is licensed under a Creative Commons Attribution 4.0 International License, which permits use, sharing, adaptation, distribution and reproduction in any medium or format, as long as you give appropriate credit to the original author(s) and the source, provide a link to the Creative Commons licence, and indicate if changes were made. The images or other third party material in this article are included in the article's Creative Commons licence, unless indicated otherwise in a credit line to the material. If material is not included in the article's Creative Commons licence and your intended use is not permitted by statutory regulation or exceeds the permitted use, you will need to obtain permission directly from the copyright holder. To view a copy of this licence, visit http://creativecommons.org/licenses/by/4.0/.

\section{References}

1. Follath F, Cleland JGF, Just H et al (2002) Efficacy and safety of intravenous levosimendan compared with dobutamine in severe low-output heart failure (the LIDO study): A randomised 
double-blind trial. Lancet 360:196-202. https://doi.org/10.1016/ S0140-6736(02)09455-2

2. Papp Z, Édes I, Fruhwald S et al (2012) Levosimendan: Molecular mechanisms and clinical implications: Consensus of experts on the mechanisms of action of levosimendan. Int J Cardiol 159:8287. https://doi.org/10.1016/J.IJCARD.2011.07.022

3. Cholley B, Caruba T, Grosjean S et al (2017) Effect of levosimendan on low cardiac output syndrome in patients with low ejection fraction undergoing coronary artery bypass grafting with cardiopulmonary bypass. JAMA 318:548. https://doi.org/10.1001/ jama.2017.9973

4. Landoni G, Lomivorotov VV, Alvaro G et al (2017) Levosimendan for hemodynamic support after cardiac surgery. N Engl J Med 376:2021-2031. https://doi.org/10.1056/NEJMoa1616325

5. Mehta RH, Leimberger JD, van Diepen S et al (2017) Levosimendan in patients with left ventricular dysfunction undergoing cardiac surgery. N Engl J Med 376:2032-2042. https://doi. org/10.1056/NEJMoa1616218

6. Putzu A, Clivio S, Belletti A, Cassina T (2018) Perioperative levosimendan in cardiac surgery: A systematic review with metaanalysis and trial sequential analysis. Int J Cardiol 251:22-31. https://doi.org/10.1016/j.ijcard.2017.10.077

7. Najjar E, Stålhberg M, Hage C et al (2018) Haemodynamic effects of levosimendan in advanced but stable chronic heart failure. ESC Hear Fail 5:302-308. https://doi.org/10.1002/ehf2.12272

8. Flett AS, Hasleton J, Cook C et al (2011) Evaluation of techniques for the quantification of myocardial scar of differing etiology using cardiac magnetic resonance. JACC Cardiovasc Imaging 4:150-156. https://doi.org/10.1016/j.jcmg.2010.11.015

9. Kim RJ, Wu E, Rafael A et al (2000) The use of contrast-enhanced magnetic resonance imaging to identify reversible myocardial dysfunction. N Engl J Med 343:1445-1453. https://doi.org/10.1056/ NEJM200011163432003

10. Becker MAJ, Cornel JH, van de Ven PM et al (2018) The prognostic value of late gadolinium-enhanced cardiac magnetic resonance imaging in nonischemic dilated cardiomyopathy. JACC Cardiovasc Imaging 11:1274-1284. https://doi.org/10.1016/j. jemg.2018.03.006

11. Aimo A, Gaggin HK, Barison A et al (2019) Imaging, biomarker, and clinical predictors of cardiac remodeling in heart failure with reduced ejection fraction. JACC Hear Fail 7:782-794. https://doi. org/10.1016/j.jchf.2019.06.004

12. Claus P, Omar AMS, Pedrizzetti G et al (2015) Tissue tracking technology for assessing cardiac mechanics: Principles, normal values, and clinical applications. JACC Cardiovasc Imaging 8:1444-1460. https://doi.org/10.1016/J.JCMG.2015.11.001

13. Nahum J, Bensaid A, Dussault C et al (2010) Impact of longitudinal myocardial deformation on the prognosis of chronic heart failure patients. Circ Cardiovasc Imaging 3:249-256. https://doi. org/10.1161/CIRCIMAGING.109.910893

14. Erley J, Genovese D, Tapaskar N et al (2019) Echocardiography and cardiovascular magnetic resonance based evaluation of myocardial strain and relationship with late gadolinium enhancement. J Cardiovasc Magn Reson 21:46. https://doi.org/10.1186/s1296 8-019-0559-y

15. Elias J, van Dongen IM, Hoebers LP et al (2020) Recovery and prognostic value of myocardial strain in ST-segment elevation myocardial infarction patients with a concurrent chronic total occlusion. Eur Radiol 30:600-608. https://doi.org/10.1007/s0033 0-019-06338-x

16. Schulz-Menger J, Bluemke DA, Bremerich J et al (2020) Standardized image interpretation and post-processing in cardiovascular magnetic resonance - 2020 update: Society for Cardiovascular Magnetic Resonance (SCMR): Board of Trustees Task Force on Standardized Post-Processing. J Cardiovasc Magn Reson 22:19. https://doi.org/10.1186/s12968-020-00610-6
17. McAlindon E, Pufulete M, Lawton C et al (2015) Quantification of infarct size and myocardium at risk: Evaluation of different techniques and its implications. Eur Heart J Cardiovasc Imaging 16:738-746. https://doi.org/10.1093/ehjci/jev001

18. Moiseyev VS, Põder P, Andrejevs N et al (2002) Safety and efficacy of a novel calcium sensitizer, levosimendan, in patients with left ventricular failure due to an acute myocardial infarction: A randomized, placebo-controlled, double-blind study (RUSSLAN). Eur Heart J 23:1422-1432. https://doi.org/10.1053/ euhj.2001.3158

19. Fredholm M, Jörgensen K, Houltz E, Ricksten SE (2018) Inotropic and lusitropic effects of levosimendan and milrinone assessed by strain echocardiography-A randomised trial. Acta Anaesthesiol Scand 62:1246-1254. https://doi.org/10.1111/aas.13170

20. Cameli M, Incampo E, Navarri R et al (2019) Effects of levosimendan in heart failure: The role of echocardiography. Echocardiography 36:1566-1572. https://doi.org/10.1111/echo.14419

21. Rocco M, Carbone I, Morelli A et al (2006) The calcium sensitizer levosimendan improves carbon monoxide poisoning related stunned myocardium: A cardiac magnetic resonance study [5]. Acta Anaesthesiol Scand 50:897-898. https://doi.org/10.111 1/j.1399-6576.2006.01052.x

22. Navarri R, Lunghetti S, Cameli M et al (2015) Neurohumoral improvement and torsional dynamics in patients with heart failure after treatment with levosimendan. IJC Hear Vasc 7:153-157. https://doi.org/10.1016/j.ijcha.2014.10.014

23. McLean AS, Huang SJ, Nalos M, Ting I (2005) Duration of the beneficial effects of levosimendan in decompensated heart failure as measured by echocardiographic indices and B-type natriuretic peptide. J Cardiovasc Pharmacol 46:830-835. https://doi. org/10.1097/01.fjc.0000189076.71730.f1

24. Wood PW, Choy JB, Nanda NC, Becher H (2014) Left ventricular ejection fraction and volumes: It depends on the imaging method. Echocardiography 31:87-100. https://doi.org/10.1111/echo.12331

25. Bellenger NG, Burgess MI, Ray SG et al (2000) Comparison of left ventricular ejection fraction and volumes in heart failure by echocardiography, radionuclide ventriculography and cardiovascular magnetic resonance. Are they interchangeable? Eur Heart J 21:1387-1396. https://doi.org/10.1053/euhj.2000.2011

26. Hoffmann R, Von Bardeleben S, Ten Cate F et al (2005) Assessment of systolic left ventricular function: A multi-centre comparison of cineventriculography, cardiac magnetic resonance imaging, unenhanced and contrast-enhanced echocardiography. Eur Heart J 26:607-616. https://doi.org/10.1093/eurheartj/ehi083

27. Riffel JH, Keller MGP, Rost F et al (2016) Left ventricular long axis strain: A new prognosticator in non-ischemic dilated cardiomyopathy? J Cardiovasc Magn Reson 18:36. https://doi. org/10.1186/s12968-016-0255-0

28. Romano S, Judd RM, Kim RJ et al (2018) Feature-tracking global longitudinal strain predicts death in a multicenter population of patients with ischemic and nonischemic dilated cardiomyopathy incremental to ejection fraction and late gadolinium enhancement. JACC Cardiovasc Imaging 11:1419-1429. https://doi. org/10.1016/j.jcmg.2017.10.024

29. Kalam K, Otahal P, Marwick TH (2014) Prognostic implications of global LV dysfunction: A systematic review and metaanalysis of global longitudinal strain and ejection fraction. Heart 100:1673-1680. https://doi.org/10.1136/heartjnl-2014-305538

30. Dalla K, Bech-Hanssen O, Ricksten SE (2019) Impact of norepinephrine on right ventricular afterload and function in septic shock-a strain echocardiography study. Acta Anaesthesiol Scand 63:1337-1345. https://doi.org/10.1111/aas.13454

31. Masci PG, Schuurman R, Andrea B et al (2013) Myocardial fibrosis as a key determinant of left ventricular remodeling in idiopathic dilated cardiomyopathy: A contrast-enhanced 
cardiovascular magnetic study. Circ Cardiovasc Imaging 6:790799. https://doi.org/10.1161/CIRCIMAGING.113.000438

32. Tayal U, Wage R, Newsome S et al (2020) Predictors of left ventricular remodelling in patients with dilated cardiomyopathy - a cardiovascular magnetic resonance study. Eur J Heart Fail:1734. https://doi.org/10.1002/ejhf.1734
Publisher's Note Springer Nature remains neutral with regard to jurisdictional claims in published maps and institutional affiliations. 